\title{
PAPER \\ Signal Detection Performance of Overlapped FFT Scheme with Additional Frames Consisting of Non-continuous Samples in Indoor Environment
}

\author{
Shoya UCHIDA ${ }^{\dagger a)}$, Akihiro SATO ${ }^{\dagger b)}$, Student Members, Mamiko INAMORI ${ }^{\dagger c)}$, \\ Yukitoshi SANADA ${ }^{\dagger \mathrm{d})}$, Members, and Mohammad GHAVAMI ${ }^{\dagger \dagger}{ }^{\dagger e}$, Nonmember
}

\begin{abstract}
SUMMARY Overlapped FFT has been proposed as a signal detection scheme in dynamic spectrum access to reduce the variance of the noise and improve the detection probability. However, the improvement of the detection probability in the conventional overlapped FFT is bounded with the upper limit of the overlap ratio. This paper proposes a new overlapped FFT scheme using additional frames. In the proposed scheme, in addition to the conventional FFT frames, new frames that consist of multiple subframes with non-continuous samples are constructed and included. It can realize the increase of the number of FFT outputs and the improvement of the detection probability compared with the conventional scheme. Numerical results through computer simulation show that the proposed scheme improves the detection probability by up to 0.07 . On indoor channel models the proposed scheme also improves the detection probability. In addition, it is clarified that as the delay spread increases the detection probability reduces due to the correlation between the frames.
\end{abstract}

key words: Cognitive radio, Overlapped FFT, Spectrum Sensing

\section{Introduction}

With the global spread of the Internet and the evolution of digital signal processing technologies in recent years, high-speed and large-capacity wireless communications have been demanded. On the other hand, the popularity of wireless communications causes the shortage of frequency spectrum available for commercial use. Therefore, cognitive radio (CR) has been attracting much attention among researchers of wireless communications [1-3]. Cognitive radio is a technology with which a mobile terminal recognizes the status of backbone networks and radio environments autonomously. One of the cognitive radio technologies is dynamic spectrum access (DSA). DSA promotes the effective use of frequency resource by finding a white space spectrum

\footnotetext{
Manuscript received January 1, 2003.

Manuscript revised January 1, 2003.

Final manuscript received January 1, 2003.

${ }^{\dagger}$ The author is with the Dept. of Electronics and Electrical Engineering, Keio University

${ }^{\dagger \dagger}$ The author is with the Dept. of Engineering and Design, London South Bank University

a) E-mail: uchida@snd.elec.keio.ac.jp

b) E-mail: asato@snd.elec.keio.ac.jp

c) E-mail: inamori@elec.keio.ac.jp

d) E-mail: sanada@elec.keio.ac.jp

e) E-mail: ghavamim@lsbu.ac.uk
}

that is temporarily not in use by a primary wireless system [4].

In the DSA, signal detection is important since the prevention of interference to a primary user is the first principle of the CR terminal. As one of signal detection schemes, overlapped FFT has been proposed [5,6]. [7] investigates the flexibility of the overlapped FFT for the rejection of the adjacent channel interference. [8] evaluates the noise reduction effect with windowing functions in the overlapped FFT.

In the overlapped FFT, the variance of the squared noise reduces in proportion to the overlap ratio and the detection probability improves as the overlap ratio increases. In other words, the improvement of the detection rate in the conventional overlapped FFT is bounded with the upper limit of the overlap ratio. This paper proposes a new overlapped FFT scheme using additional frames. In the proposed scheme, in addition to the conventional FFT frames, new frames are constructed and included in order to increase the number of FFT outputs. To reduce the correlation to the conventional frames, the additional frames consist of multiple subframes with non-continuous samples.

This paper is organized as follows. Firstly, signal detection with the conventional overlapped FFT and the proposed scheme is described in Section 2. The probability of detection $\left(P_{D}\right)$ and the probability of false alarm $\left(P_{F A}\right)$ using the conventional and proposed schemes are evaluated in Section 3. Finally, our conclusions are presented in Section 4.

\section{System Model}

\subsection{Conventional Overlapped FFT}

The signal detection scheme with the conventional overlapped FFT is presented in Figs. 1 and 2. The received signal $s(t)$ is given as

$$
s(t)=h(t) \otimes x(t)+n(t)
$$

where $h(t)$ is the impulse response of the channel between the input of the transmit filter and the output of the receive filter, $x(t)$ is the baseband signal, $n(t)$ is 


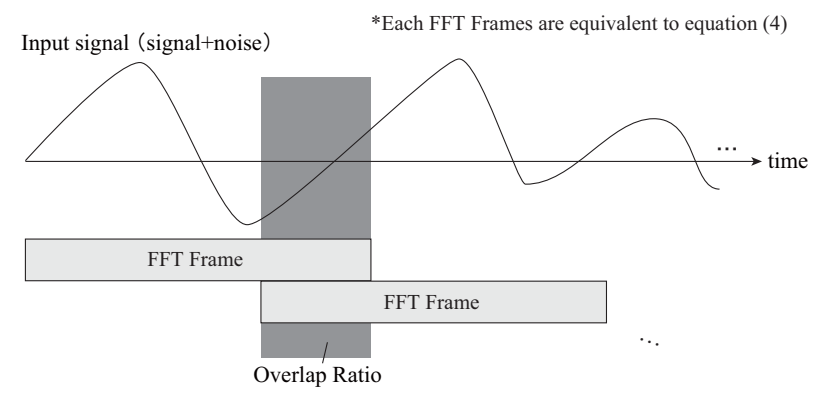

Fig. 1 Frame construction in conventional overlapped FFT.

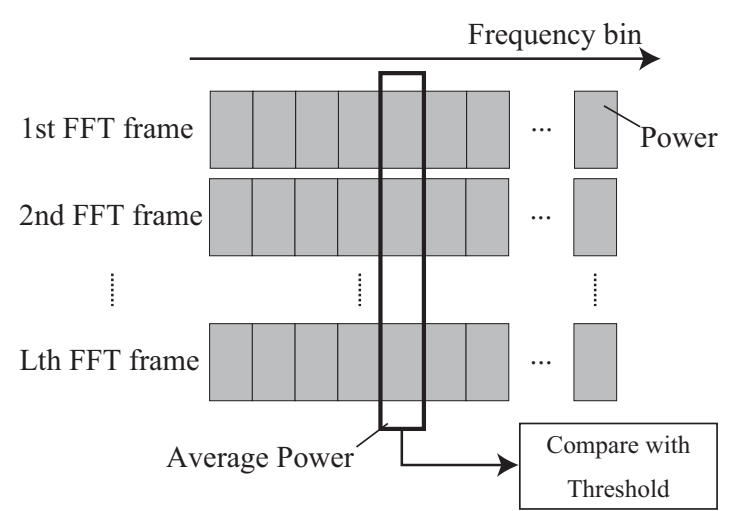

Fig. 2 Signal detection with overlapped FFT.

the additive white Gaussian noise (AWGN), and $\otimes$ denotes convolution. The impulse response of the channel is given by

$$
h(t)=p_{r x}(t) \otimes c(t) \otimes p_{t x}(t)
$$

where $p_{t x}(t)$ and $p_{r x}(t)$ are the impulse responses of the transmit and receive filters, and $c(t)$ is the impulse response of the multipath channel. Subsequently, the received signal $s(t)$ is converted into digital samples as $s[n]=s\left(n T_{s}\right)$ through an analog-to-digital converter (ADC) where $n(n=0,1, \ldots)$ is the time index and $T_{s}$ is the sampling interval. The number of FFT outputs is given as

$$
L_{c}=\left\lfloor\frac{I-N}{N-d_{O L}}\right\rfloor+1
$$

where $L_{c}$ is the number of the FFT frames, $I$ is the number of the samples, $N$ is the FFT size, $d_{O L}$ is the number of the samples overlapping between 2 consecutive frames and $\lfloor *\rfloor$ implies the minimum integer more than $*$. The number of FFT outputs increases as the overlap ratio $\left(d_{o l}=d_{O L} / N\right)$ grows. Consequently, the variance of the squared and averaged FFT outputs decreases.

The output of the $l_{c}$ th FFT frame in the $k$ th bin is given as

$$
S_{l_{c}}[k]=\sum_{n=0}^{N-1} s\left[N\left(l_{c}-1\right)\left(1-d_{o l}\right)+n\right] \exp \left(-j \frac{2 \pi n k}{N}\right)
$$

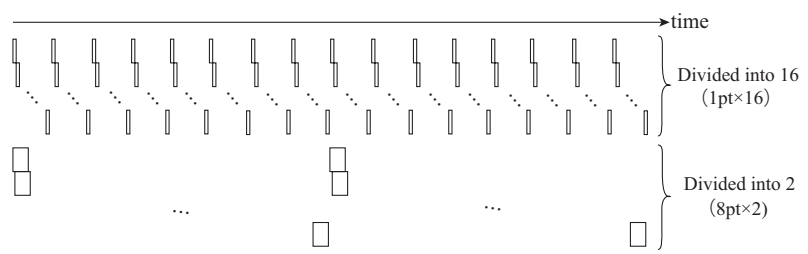

Fig. 3 Frame construction in proposed overlapped FFT.

where $N$ is the FFT size and $d_{o l}$ is the overlap ratio between 2 consecutive frames. Finally, the average energy in each bin is calculated as

$$
|S[k]|^{2}=\frac{\sum_{l_{c}=1}^{L}\left|S_{l_{c}}[k]\right|^{2}}{L_{c}}
$$

where $L_{c}$ is the number of the FFT frames.

\subsection{Proposed Overlapped FFT}

In this section, the proposed overlapped FFT scheme using additional subframes are explained. The theoretical analysis on the variance of the squared noise averaged over the FFT outputs and the circuit size of the proposed scheme are also presented.

\subsubsection{Frame Format}

The proposed scheme includes additional FFT frames to reduce the variance of the squared noise by increasing the number of the FFT outputs for averaging. The new frames are constructed from the same received signal. However, those frames consist of multiple subframes that come from the different parts of the received signal. This is to reduce the correlation between the additional frames and the conventional frames.

Fig. 3 shows the two different ways to collect the samples for the new frames. One way is to divide the frame to small subframes and each subframe picks up a single sample from the received signal. The other one divides it into only 2 subframes. In this case each subframe is formed with the consecutive samples. These two different formats of the subframes make a large difference on the frequency spectrum of the received signal at the FFT outputs.

The frequency responses of the transmit filter employed in computer simulation with the 2 different frame formats are shown in Fig. 4. The size of the FFT is set to 16 . If the frame is divided into 2 subframes, the frequency response is close to the original. On the other hand, if it is divided into 16 subframes, no suppression in the out-of-band signal can be expected. This causes the deterioration of the signal detection probability. Thus, the proposed scheme uses the FFT frames with 2 subframes.

\subsubsection{Proposed Overlapped FFT}

The signal detection with the proposed overlapped FFT 


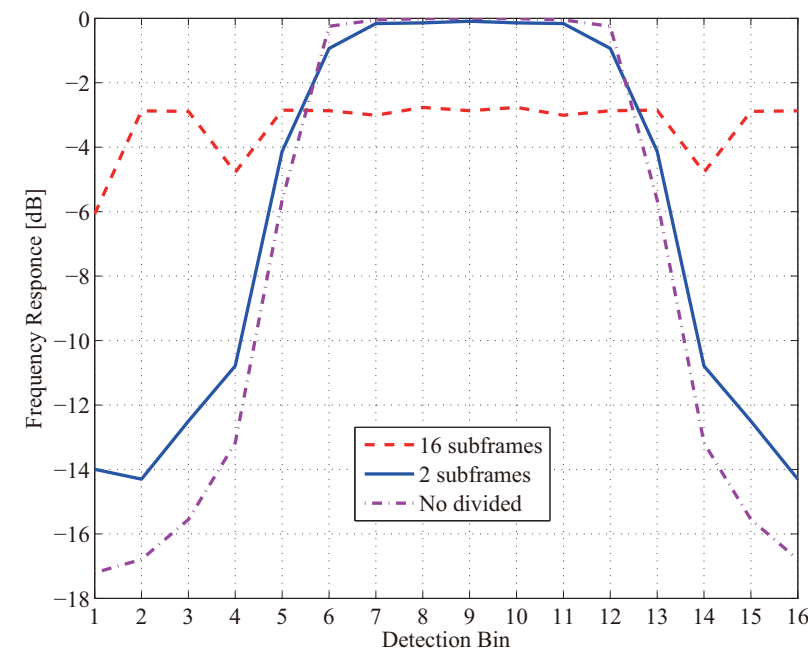

Fig. 4 Difference in frequency response by the number of subframes.

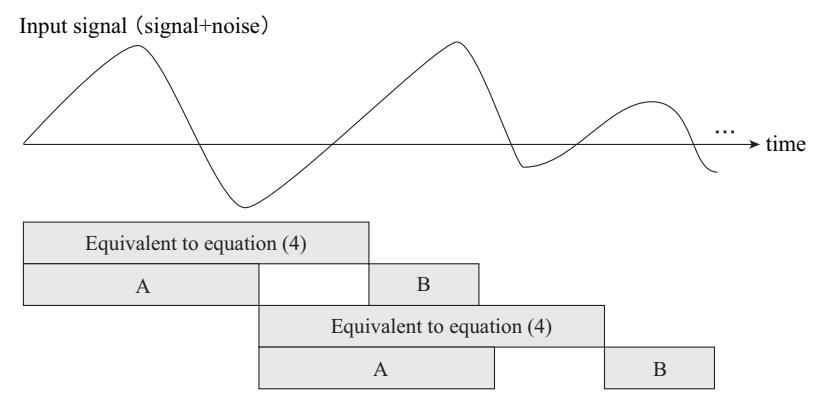

Fig. 5 Frame placement of the proposed scheme.

scheme is presented in Fig. 5. In addition to the conventional frames, new FFT frames are used to increase the FFT outputs. The new frames are constructed as shown in Fig. 5. The first subframe (A) overlaps with the part of the conventional frame while the second subframe (B) consists of the samples just after the first conventional subframe. In this way, the correlation between the conventional and the additional frames can be controlled with the length of the first subframe.

Therefore, the output on the $k$ th FFT bin for the $l_{s}$ th additional frame is given as

$$
\begin{aligned}
& S_{l_{s}}[k] \\
& =\sum_{i=0}^{N-d_{o l}-1} s\left[N\left(l_{s}-1\right)\left(1-d_{o l}\right)+i\right] \times \exp \left(-j \frac{2 \pi i k}{N}\right) \\
& +\sum_{i=N-d_{o l}}^{N-1} s\left[N\left(l_{s}-1\right)\left(1-d_{o l}\right)+N+\left(i-N-d_{o l}-1\right)\right] \\
& \quad \times \quad \exp \left(-j \frac{2 \pi i k}{N}\right)
\end{aligned}
$$

where $N$ is the FFT size and $d_{o l}$ is the overlap ratio of the conventional frames. In Eq. (6), the first term cor- responds to the first subframe (A) and the second term calculates the FFT output for the second subframe (B).

Finally, the average energy on the $k$ th bin is calculated as

$$
|S[k]|^{2}=\frac{\sum_{l_{c}=1}^{L_{c}}\left|S_{l_{c}}[k]\right|^{2}+\sum_{l_{s}=1}^{L_{s}}\left|S_{l_{s}}[k]\right|^{2}}{L_{c}+L_{s}}
$$

where $L_{c}$ and $L_{s}$ are the number of the FFT outputs of the conventional and additional frames.

\subsection{Theoretical Analysis on Variance of Squared Noise}

In this section the variance of the squared noise averaged over the FFT outputs is theoretically evaluated. The following theoretical analysis assumes that the input of the detector only contains the noise. The output of the detector in each FFT bin follows chi-square distribution [9]. If the total number of the frames $L\left(=L_{c}+L_{s}\right)$ increases, the output after averaging approaches to correlated Gaussian distribution due to the law of great numbers [9]. The joint probability density function $(\mathrm{PDF})$ is given as

$$
\begin{aligned}
& p\left(S_{1}[k], S_{2}[k], \ldots, S_{n}[k]\right) \\
& =\frac{1}{(2 \pi)^{L / 2}[\operatorname{det} \mathbf{R}[k]]^{1 / 2}} \exp \left[-\frac{1}{2} \mathbf{S}[k]^{T} \mathbf{R}[k]^{-1} \mathbf{S}[k]\right]
\end{aligned}
$$

where $p\left(S_{1}[k], S_{2}[k], \ldots, S_{n}[k]\right)$ is the joint PDF of the FFT outputs in the $k$ th bin, $\mathbf{S}=\left[S_{1}[k] S_{2}[k] \ldots S_{L}[k]\right]^{T}$, and $\mathbf{R}[k]$ is the correlation matrix of the FFT outputs in the $k$ th bin, which is given as

$$
\mathbf{R}[k]=\left(\begin{array}{cccc}
\gamma_{1,1}[k] & \gamma_{1,2}[k] & \ldots & \gamma_{1, L}[k] \\
\gamma_{2,1}[k] & \gamma_{2,2}[k] & \ldots & \gamma_{2, L}[k] \\
\vdots & \vdots & \ddots & \vdots \\
\gamma_{L, 1}[k] & \gamma_{L, 2}[k] & \ldots & \gamma_{L, L}[k]
\end{array}\right)
$$

where $\gamma_{i, j}$ is the correlation coefficient between the $i$ th and $j$ th frames. This correlation matrix is a Hermitian matrix and the eigenvalues are real values [10]. The eigenvalues of $\mathbf{R}[k]$ are defined as

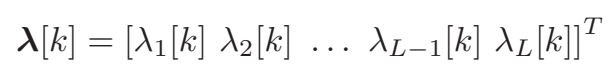

where $\lambda_{l}$ is the $l$ th eigenvalue. Finally, the sum of the outputs from the detector is approximated by Gaussian distribution with the following average and variance,

$$
\begin{gathered}
\text { Average : } \sum_{l=1}^{L} \lambda_{l}[k] \sigma^{2}, \\
\text { Variance : } \sum_{l=1}^{L} 2 \lambda_{l}[k]^{2} \sigma^{4},
\end{gathered}
$$

where $\sigma^{2}$ is the variance of the noise, $n(t)$. In computer simulation, the threshold for signal detection is determined with the Gaussian approximation so that $P_{F A}$ satisfies a predetermined value. 


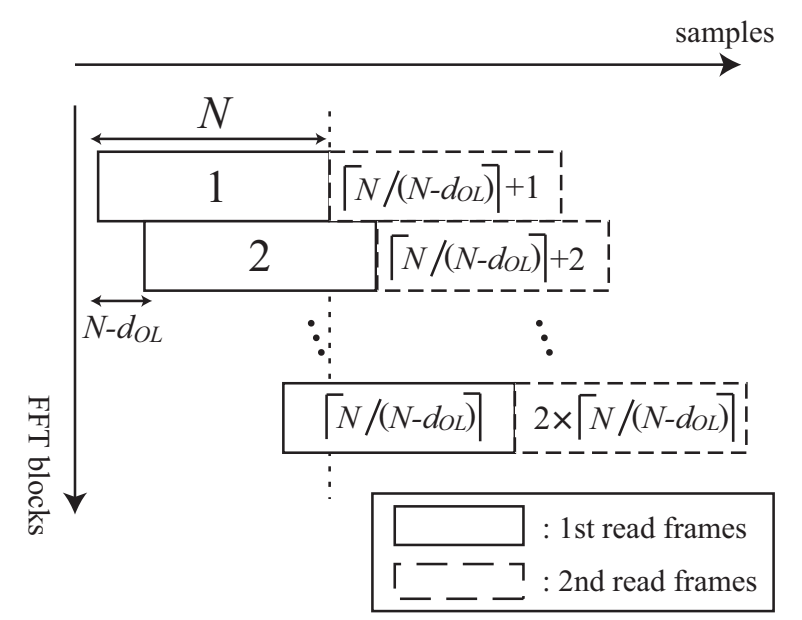

Fig. 6 FFT Blocks of the Conventional Scheme.

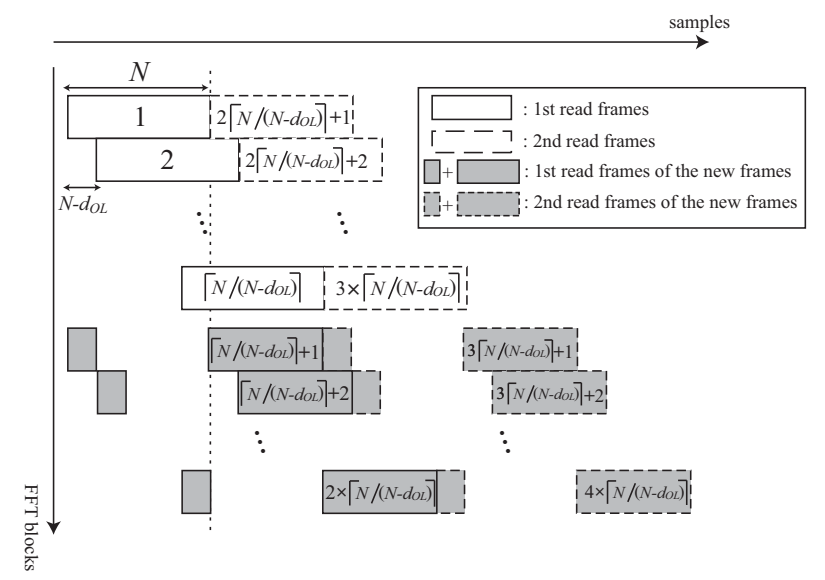

Fig. 7 FFT Blocks of the Proposed Scheme.

The eigenvalues of the Hermitian matrix have a relationship to the Frobenius norm of the matrix as follows [10]:

$$
\begin{aligned}
\|\mathbf{R}[k]\|_{F} & =\sqrt{\sum_{i=1}^{L} \sum_{j=1}^{L}\left|\gamma_{i, j}[k]\right|} \\
& =\sqrt{\operatorname{tr}(\mathbf{R}[k] * \mathbf{R}[k])}=\sqrt{\sum_{l=1}^{L} \lambda_{l}[k]^{2}}
\end{aligned}
$$

Thus, Eq. (12) can be rewritten as

$$
\text { Variance : } 2 \sum_{i=1}^{L} \sum_{j=1}^{L}\left|\gamma_{i, j}[k]\right| \sigma^{4} \text {. }
$$

Therefore, as the correlation between the frames reduces, the variance of the squared noise after averaging decreases.

\subsection{Circuit Size}

The number of FFT frames per 1 frame duration in
Table 1 Simulation Conditions

\begin{tabular}{|c|c|}
\hline Modulation & QPSK / OFDM \\
\hline Number of subcarriers & 64 \\
\hline Number of data subcarriers & 52 \\
\hline Transmit filter & $\begin{array}{c}\text { Fourier transform } \\
\text { of the spectrum mask } \\
\text { with hamming window }\end{array}$ \\
\hline Filter length & $50 T_{s}$ \\
\hline Filter bandwidth & $20 \mathrm{MHz}$ \\
\hline Channel & $\begin{array}{c}\text { AWGN } \\
\text { Indoor Residential-A } \\
\text { Indoor Office-B }\end{array}$ \\
\hline Observation period & $24 \mu \mathrm{s}$ \\
\hline Sampling rate & $40 \mathrm{MHz}$ \\
\hline FFT size & 16 \\
\hline Window function & Hamming window \\
\hline$P_{F A}$ & $0.1,0.01$ \\
\hline Detection bin & 8 \\
\hline Number of trials & $10^{5}$ \\
\hline
\end{tabular}

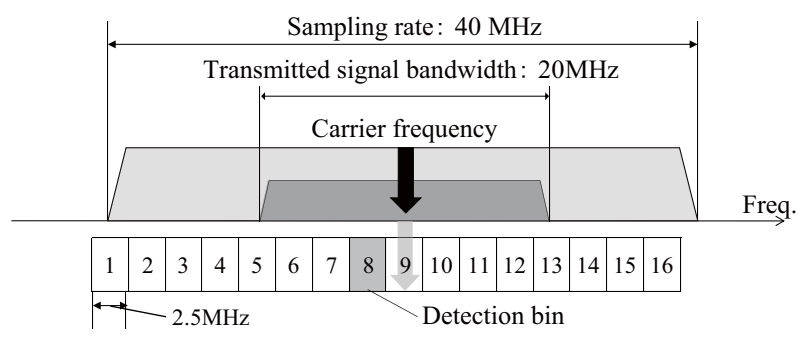

Fig. 8 Relationship between bandwidth and detection bin.

average is $\left\lceil N /\left(N-d_{O L}\right)\right\rceil$ in the conventional scheme and $2\left\lceil N /\left(N-d_{O L}\right)\right\rceil$ in the proposed scheme as shown in Figs. 6 and 7 where $\lceil *\rceil$ means the maximum integer less than $*$. Thus, if the processing speed of FFT is the same as the frame duration, $\left\lceil N /\left(N-d_{O L}\right)\right\rceil$ FFT blocks and $2\left\lceil N /\left(N-d_{O L}\right)\right\rceil$ FFT blocks are required for real time signal sensing in the conventional and proposed schemes. Since an $802.11 \mathrm{a} / \mathrm{g}$ WLAN receiver has an FFT block with the size of $64,(64 / N)$ FFT subblocks with the size of $N$ are available. Thus, the circuit size in terms of the number of FFT blocks increases $\lceil N /(N-$ $\left.\left.d_{O L}\right)\right\rceil \times(N / 64)$ times or $2\left\lceil N /\left(N-d_{O L}\right)\right\rceil \times(N / 64)$ times in the conventional and proposed schemes.

\section{Numerical Results}

\subsection{Simulation Conditions}

Table 1 shows the simulation conditions. The transmit signal follows the format of the IEEE $802.11 \mathrm{~g}$ standard. The symbols are modulated with QPSK on each subcarrier. The number of the subcarriers and the number of the data subcarriers are 64 and 52, respectively. The tap coefficients of the transmit filter are calculated from the $11 \mathrm{~g}$ spectrum mask and a hamming window is multiplied to the inverse Fourier transform of the spectrum mask. The filter length is $50 T_{s}$ and the bandwidth is 20 $\mathrm{MHz}$. As channel models, an AWGN channel as well as 


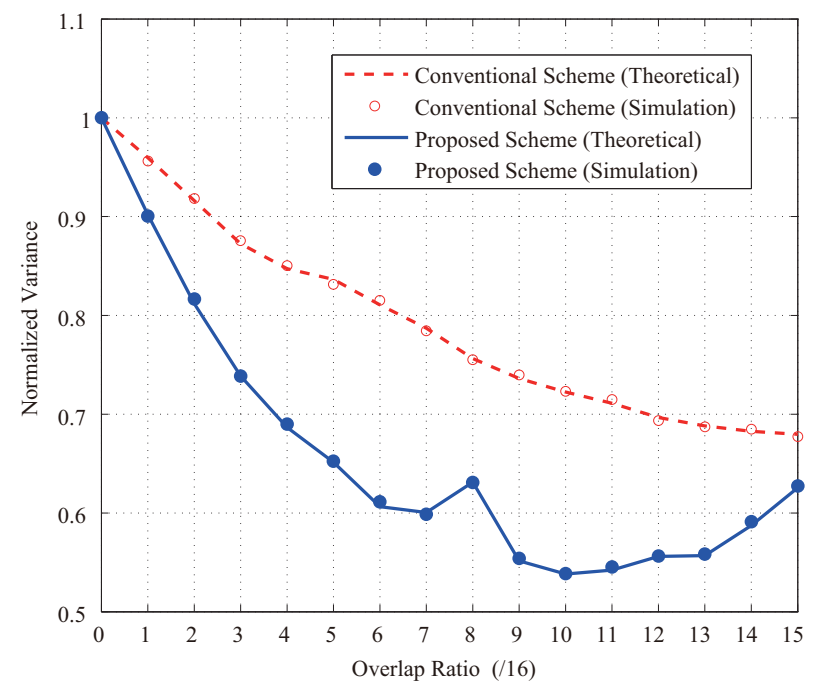

Fig. 9 Normalized variance vs. overlap ratio.

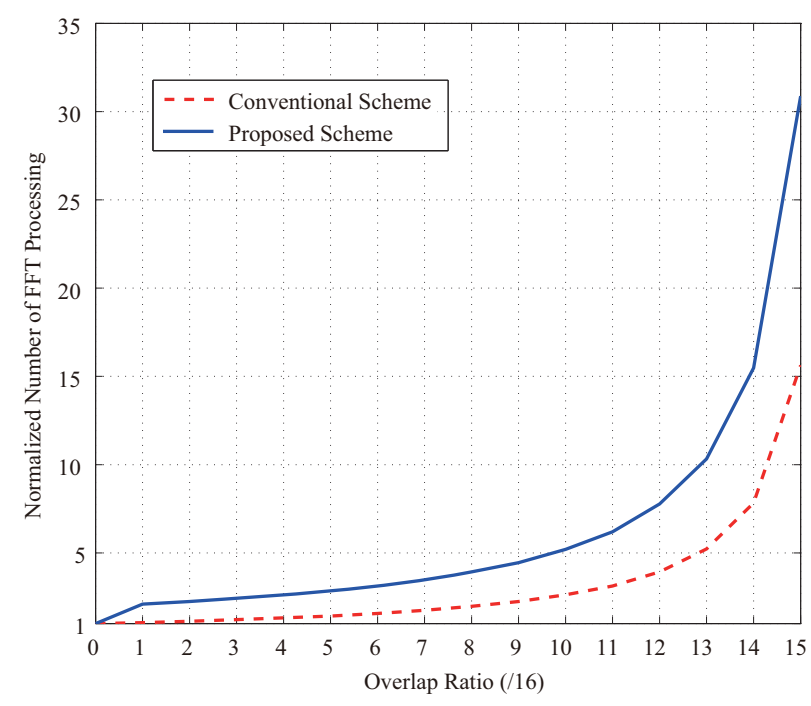

Fig. 10 Number of FFT processing vs. overlap ratio.

multipath fading channel models, Indoor Residential-A and Indoor Office-B, are assumed [11]. As for the receiver, the observation period for signal sensing is set to $24 \mu \mathrm{s}$. The sample rate is $40 \mathrm{MHz}$ and the size of FFT is 16 . $P_{F A}$ is set to 0.1 or 0.01 [12]. The 8 th detection bin is evaluated while the carrier frequency is located in the middle of the 9th bin as shown in Fig. 8. The number of trials is set to $10^{5}$ for the numerical results.

\subsection{Variance of the FFT Outputs without Signal}

Figs. 9 and 10 show the relationship between the variance of the FFT outputs only with the noise and the overlap ratio or the number of FFT processing. For comparison, the variance of the squared noise after averaging is normalized by that without overlapping. The number of FFT processing is normalized by $M / N$,
Table 2 Number of FFT Processing

\begin{tabular}{|c|c|c|}
\hline & $\begin{array}{c}\text { Number of } \\
\text { FFT Processing }\end{array}$ & Overlap Ratio \\
\hline Proposed Scheme & 314 & $10 / 16$ \\
\hline Conventional Scheme & 315 & $13 / 16$ \\
\hline Conventional Scheme & 945 & $15 / 16$ \\
\hline
\end{tabular}

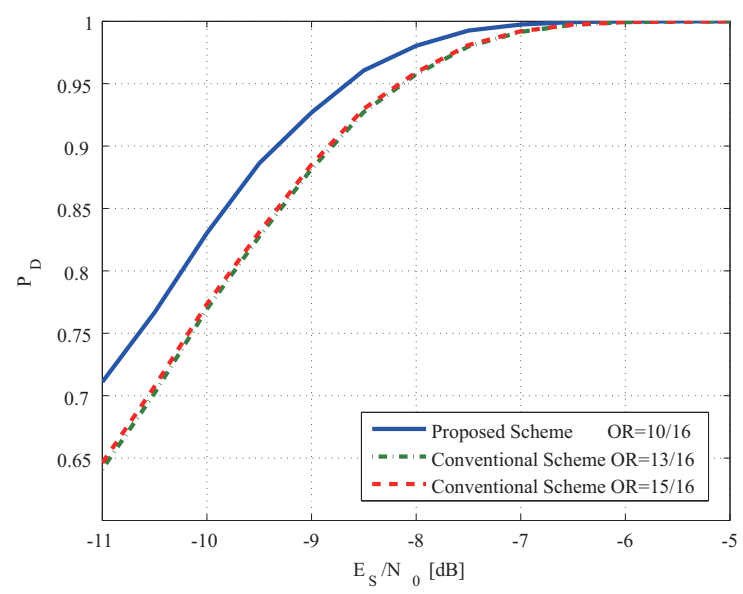

Fig. 11 Probability of detection vs. SNR $\left(P_{F A}=0.1\right)$, AWGN channel.

where $M$ is the number of the samples and $N$ is the size of FFT.

The variance of the squared FFT outputs only with the noise is shown in Fig. 9. The variance is normalized by the value without overlapping. As compared to the conventional scheme, the proposed scheme reduces the variance by about 0.15 . This is because of the increase of the number of the FFT frames. The variance reduces monotonically as the overlap ratio increases in the conventional scheme. On the other hand, the proposed scheme shows the smallest value at the overlap ratio of $10 / 16$. When the overlap ratio increases, the total value of the power of the correlation coefficients in Eq. (14) increases and the variance also grows. Meanwhile, the overlap ratio of 0.5 also increases the total sum of the power of the correlation coefficients. Thus, the variance has the peak at the overlap ratio of 0.5 .

With regards to Fig. 9, in the following simulations we assume that the overlap ratio is $10 / 16$ because it shows the minimum variance. For the comparison, the overlap ratio of $13 / 16$ is chosen for the conventional scheme since the number of FFT processing is almost the same as that of the proposed scheme, as shown in Table 2. It should be mentioned that, the overlap ratio of 15/16 has also been evaluated and has given the smallest variance in the conventional scheme.

\subsection{Detection Probability on AWGN Channel}

The numerical results on the AWGN channel are shown in this section. The probability of false alarm, $P_{F A}$, is 


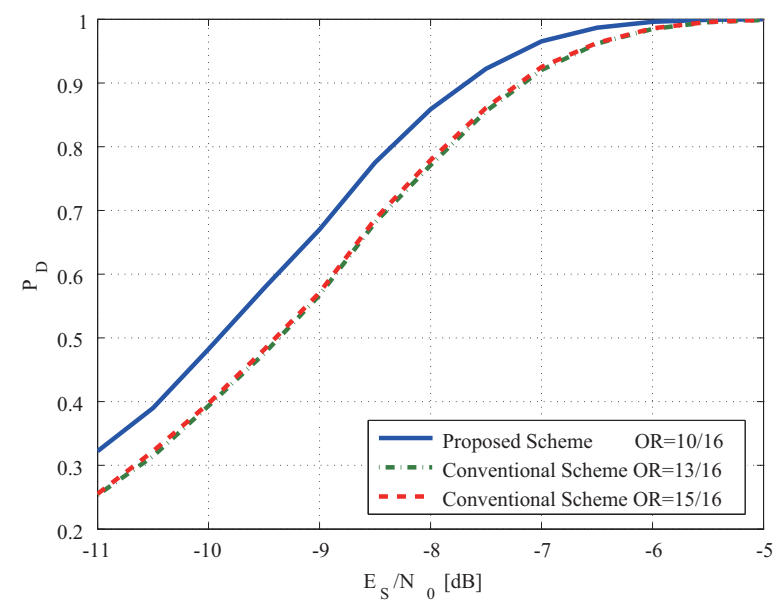

Fig. 12 Probability of detection vs. SNR $\left(P_{F A}=0.01\right)$, AWGN channel.

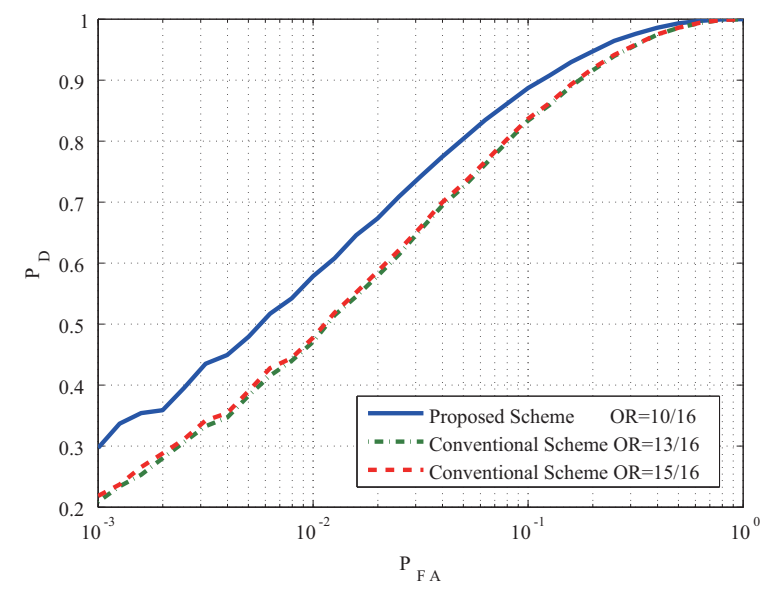

Fig. 13 ROC at $E_{s} / N_{0}=-9.5 \mathrm{~dB}$, AWGN channel.

fixed to 0.1 or 0.01 . $E_{s} / N_{0}$ ranges from -11 to $-5 \mathrm{~dB}$ where $E_{s}$ is the energy of the signal per symbol and $N_{0}$ is the noise spectrum density.

Figs. 11 and 12 show the probability of detection, $P_{D}$, versus the signal-to-noise ratio (SNR) for $P_{F A}$ of 0.1 or 0.01 . The detection probability of the proposed scheme is better than that of the conventional scheme on the whole range of $E_{s} / N_{0}$. In particular, the proposed scheme improves the detection probability by about 0.06 or 0.07 at $P_{D}=0.9$.

Fig. 13 shows the receiver operating characteristic (ROC) curves for $E_{s} / N_{0}$ of $-9.5 \mathrm{~dB}$. The ROC also improves with the proposed scheme. When $P_{D}$ of the proposed scheme is 0.9 , it is 0.05 higher than the probabilities with the conventional scheme.

3.4 Detection Probability on Multipath Fading Channel Models

The numerical results on multipath fading channel models are shown in this section.

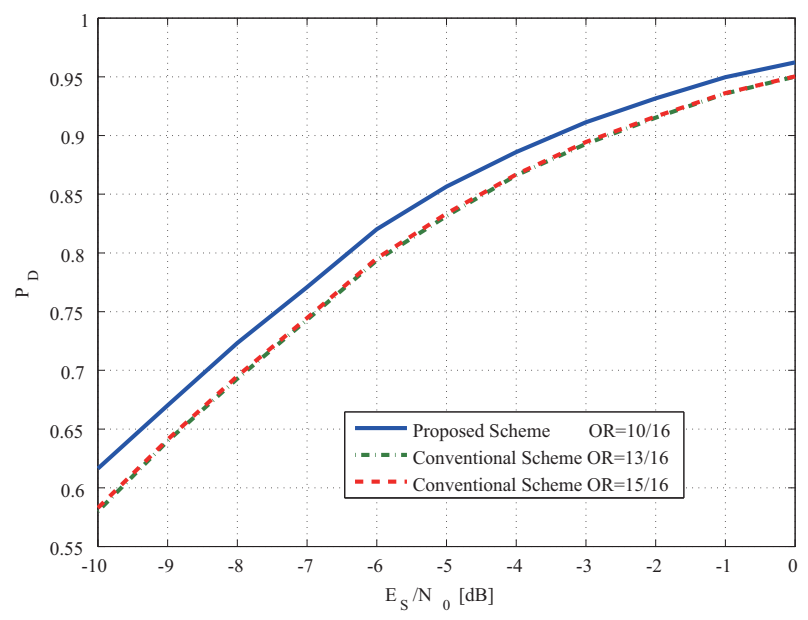

Fig. 14 Probability of detection vs. SNR $\left(P_{F A}=0.1\right)$, Indoor Residential-A channel model.

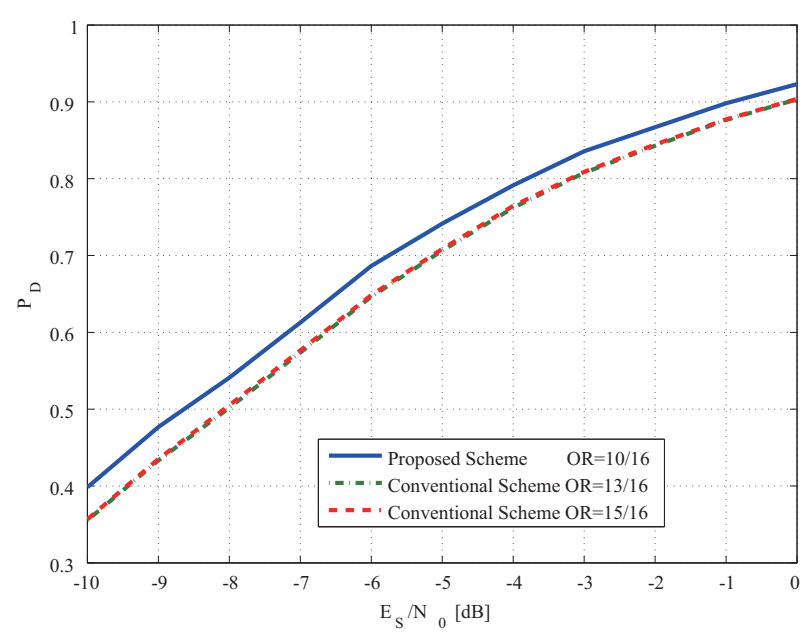

Fig. 15 Probability of detection vs. SNR $\left(P_{F A}=0.01\right)$, Indoor Residential-A channel model.

Figs. 14 and 15 show $P_{D}$ versus SNR for $P_{F A}$ of 0.1 or 0.01 on the Indoor Residential-A channel model. $E_{s} / N_{0}$ ranges from -10 to $0 \mathrm{~dB}$. The detection probability of the proposed scheme is better than that of the conventional scheme on the whole range of $E_{s} / N_{0}$. In particular, the proposed scheme improves the detection probability by about 0.03 at $P_{D}=0.9$.

Fig. 16 shows the ROC curves for $E_{s} / N_{0}$ of -3 $\mathrm{dB}$. The value of $E_{s} / N_{0}$ is fixed to the value when $P_{F A}$ is fixed to 0.1 and $P_{D}$ of the proposed scheme becomes 0.9. The ROC also improves with the proposed scheme. When $P_{D}$ of the proposed scheme is 0.9 , it is 0.03 higher than the probabilities with the conventional scheme.

3.5 Comparison of Indoor Residential-A and Indoor Office-B Channel Model

In this paper, the performance on the Indoor Residential-A channel model and the Indoor Office-B 


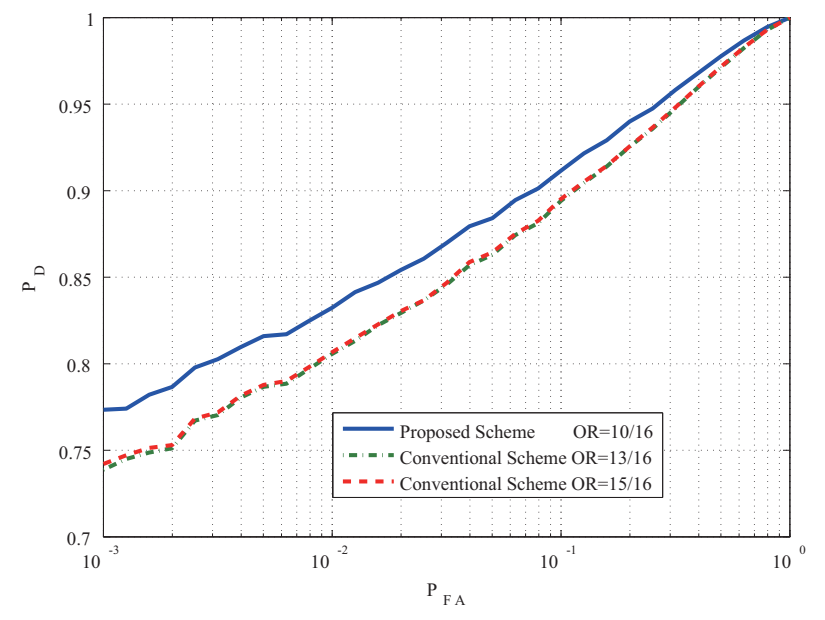

Fig. 16 ROC under $E_{s} / N_{0}=-3 \mathrm{~dB}$, Indoor Residential-A channel model.

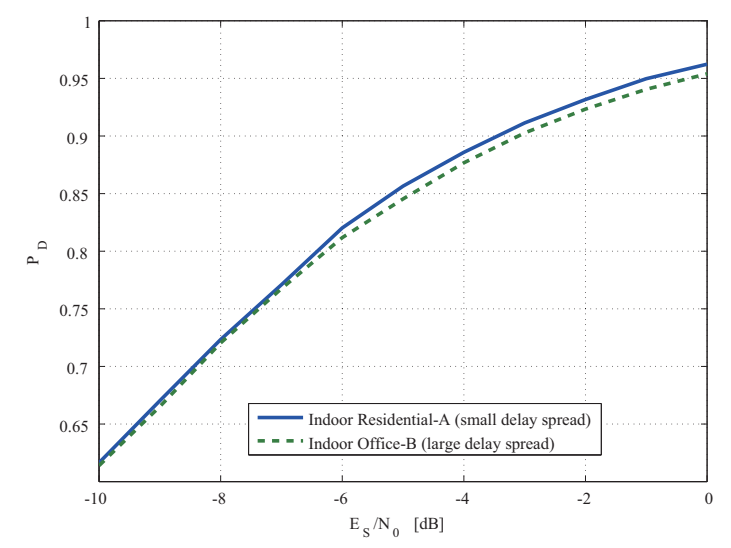

Fig. 17 Probability of detection vs. SNR $\left(P_{F A}=0.1\right)$, comparison of Indoor Residential-A channel model and Indoor OfficeB channel model.

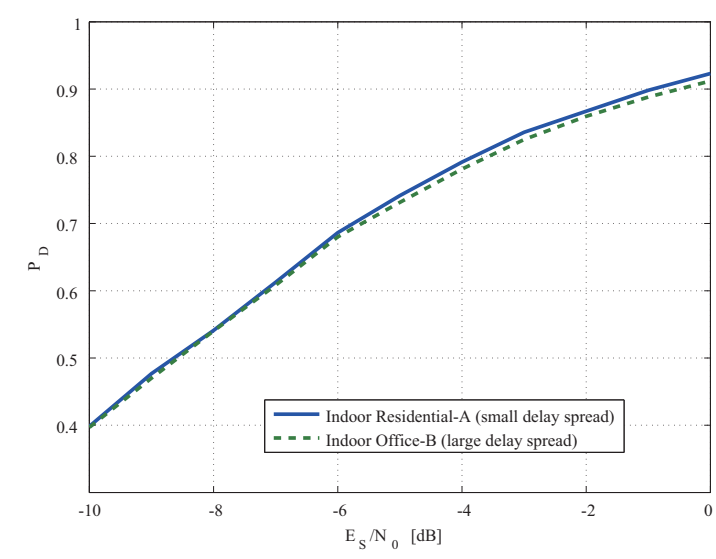

Fig. 18 Probability of detection vs. SNR $\left(P_{F A}=0.01\right)$, comparison of Indoor Residential-A channel model and Indoor OfficeB channel model.

channel model have been evaluated. The same tendency of the performance curves can be observed on both of the channel models. The slight difference in

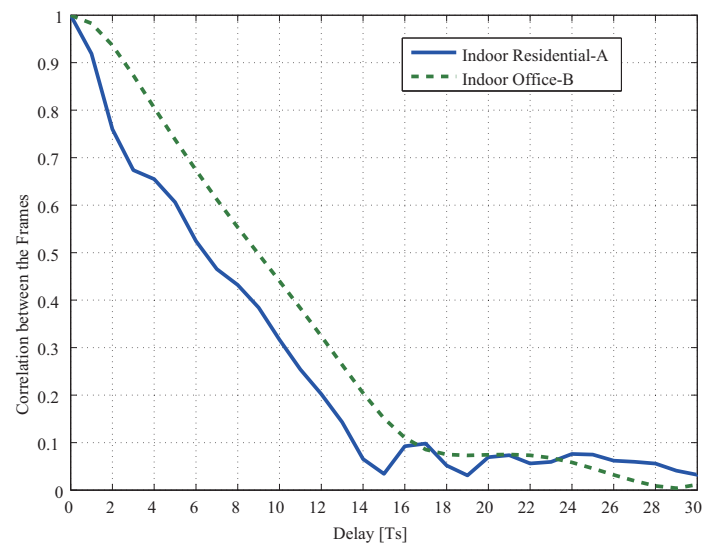

Fig. 19 Amount of delay vs. correlation between the frames (8th bin).

the detection probability is found between those channel models. Figs. 17 and 18 show $P_{D}$ versus the SNR for $P_{F A}$ of 0.1 or 0.01 . The detection probability on the Indoor Office-B channel model is degraded more than that on the Indoor Residential-A channel model. It is caused by the difference of the correlation between the frames on those two channel models.

Fig. 19 shows the amount of delay versus the correlation between the frames for the 8 th bin on those channel models. The correlation on the Indoor OfficeB channel model is higher than that on the Indoor Residential-A channel model at the delay of less than $16 T_{s}$. This is because the delay spread of the Indoor Office-B channel model is larger. As a result, the correlation between the frames increases and the larger correlation degrades the detection probability on the Indoor Office-B channel model.

\section{Conclusions}

In this paper, the overlap FFT scheme for signal sensing with the additional frames has been proposed. In the proposed scheme in addition to the conventional frames, the new frames are constructed to increase the total number of the FFT outputs. The additional frames are formed with the subframes that consist of the different part of the received samples to reduce the correlation to the conventional frames.

The variances of the power of the FFT outputs without signal are theoretically analyzed for the conventional and proposed schemes. When there is no signal, the variance of the power of the FFT outputs reduces in proportion to the overlap ratio in the conventional scheme while there is the minimum point in the proposed scheme.

By increasing the number of the frames the proposed scheme improves the probability of detection by about 0.07 .

It has also been clarified that on the Indoor Residential-A channel model and the Indoor Office- 
B channel model the proposed scheme improves the probability of detection by about 0.03 . In addition, as the delay spread increases, the detection probability reduces due to the correlation between the frames.

\section{References}

[1] J. Mitra III , G. Q. Maguire Jr., "Cognitive Radio: Making Software Radios More Personal," IEEE Personal Communications, vol. PC-6, pp. 13-18, Aug. 1999.

[2] J. Mitra III, "Cognitive Radio for Flexible Mobile Multimedia Communications," 1999 IEEE International Workshop on Mobile Multimedia Communications, pp. 3-10, Nov. 1999.

[3] S. Haykin, "Cognitive Radio: Brain-Empowered Wireless Communications," IEEE Journal on Selected Areas in Communications, vol. 23, no. 2, pp. 201-220, Feb. 2005.

[4] Q. Zhao, B. M. Sadler, "A Survey of Dynamic Spectrum Access," IEEE Signal Processing Magazine, vol. 24, Issue 3, pp. 79-89, May 2007.

[5] B. H. Maranda, "On the False Alarm Probability for an Overlapped FFT Processor," IEEE Trans. on Aerospace and Electronic System, vol. 32, no. 4, pp. 1452-1456, Oct. 1996.

[6] H. Fan, S. Wang, F. Chen, Y. Zhu, "Frame-overlapped Zoom-FFT Optimization in PD Radar Application," International Conference on Radar, pp. 1-5, Oct. 2006.

[7] S. Wang, R. Inkol, S. Rajan, F. Patenaude, "Detection of Narrow-Band Signals through the FFT and Polyphase FFT Filter Banks: Noncoherent versus Coherent Integration," IEEE Trans. on Instrumentation and Measurement, vol. 59, no. 5, pp. 1424-1438, May 2010.

[8] T. Tomioka, T. Tomizawa, T. Kobayashi, "High-Sensitivity Carrier Sensing using Overlapped FFT for Cognitive Radio Transceivers," IEEE 69th Vehicular Technology Conference, Apr. 2009.

[9] J. Proakis, Digital Communications, 2nd Edition, New York: McGraw-Hill, 1989.

[10] Gene H. Golub, Charles F. Van Loan , Matrix Computations, 3rd Edition, The Johns Hopkins University Press, 1996.

[11] Joint Technical Committee of Committee T1 R1P1.4 and TIATR46.3.3/TR45.4.4 on Wireless Access, "Draft Final Report on RF Channel Characterization," Paper No. JTC(AIR)/94. 01. 17-238R4, Jan. 1994.

[12] IEEE 802.22-Part 22: Cognitive Wireless RAN Medium Access Control (MAC) and Physical Layer (PHY) Specifications: Policies and Procedures for Operation in the TV Bands

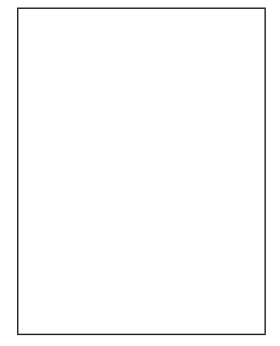

Akihiro Sato was born in Kagawa, Japan in 1988. He received his B.E. degree in electronics engineering from Keio University, Japan in 2011. Since April 2011, he has been a graduate student in School of Integrated Design Engineering, Graduate School of Science and Technology, Keio University. His research interests are mainly concentrated on software defined radio.

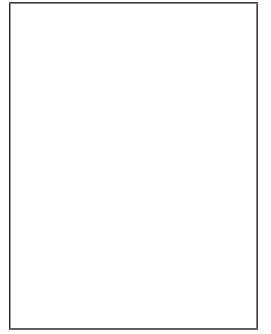

Mamiko Inamori was born in Kagoshima, Japan in 1982. She received her B.E. and M.E. degrees in electronics engineering from Keio University, Japan in 2005 and 2007 respectively. Since April 2007, she has been a Ph.D. candidate in School of Integrated Design Engineering, Graduate School of Science and Technology, Keio University. Her research interests are mainly concentrated on software defined radio.

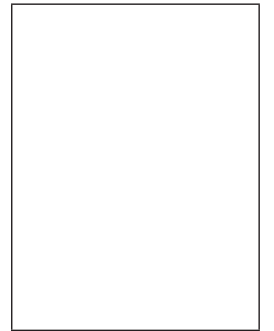

Yukitoshi Sanada was born in Tokyo in 1969. He received his B.E. degree in electrical engineering from Keio University, Yokohama Japan, his M.A.Sc. degree in electrical engineering from the University of Victoria, B.C., Canada, and his $\mathrm{Ph} . \mathrm{D}$. degree in electrical engineering from Keio University, Yokohama Japan, in 1992, 1995, and 1997, respectively. In 1997 he joined the Faculty of Engineering, Tokyo Institute of Technology as a Research Associate. In 2000 he joined Advanced Telecommunication Laboratory, Sony Computer Science Laboratories, Inc, as an associate researcher. In 2001 he joined Faculty of Science and Engineering, Keio University, where he is now a professor. He received the Young Engineer Award from IEICE Japan in 1997. His current research interest are in software defined radio, cognitive radio, and OFDM systems.

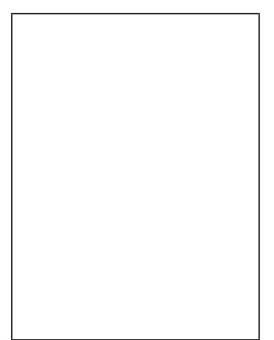

Mohammad Ghavami is a professor of Telecommunications Engineering at London South Bank University, UK. He is the leading author of the book "Ultra Wideband Signals and Systems" and has published over 120 technical papers in major international journals and conferences on areas related to ultra wideband systems, smart antenna, adaptive filters and biomedical signal processing. He completed his Ph.D. degree with Great Distinction in electrical engineering in 1993. During his Ph.D. studies he was awarded the DAAD scholarship at the University of Kaiserslautern, Germany from 1990 to 1993. From 1998 to 2000 he was a JSPS postdoctoral fellow at the Yokohama National University, Japan. From 2000 to 2002 he was a researcher at the Sony Computer Science Laboratories in Tokyo, Japan. He has won two invention awards from Sony Ltd. 